\title{
Necrotizing pancreatitis: challenges and solutions
}

This article was published in the following Dove Press journal:

Clinical and Experimental Gastroenterology

31 October 2016

Number of times this article has been viewed

\section{Victoria A Bendersky' \\ Mohan K Mallipeddi² \\ Alexander Perez ${ }^{2}$ \\ Theodore N Pappas ${ }^{2}$}

'School of Medicine, ${ }^{2}$ Department of Surgery, Duke University, Durham, NC, USA
Correspondence: Theodore N Pappas

Department of Surgery, Duke

University, DUMC Box 3479,

Durham, NC 277I0, USA

$\mathrm{Tel}+\mathrm{I} 91968 \mathrm{I} 3442$

Fax +19196681826

Email theodore.pappas@duke.edu
Abstract: Acute pancreatitis is a common disease that can progress to gland necrosis, which imposes significant risk of morbidity and mortality. In general, the treatment for pancreatitis is a supportive therapy. However, there are several reasons to escalate to surgery or another intervention. This review discusses the pathophysiology as well as medical and interventional management of necrotizing pancreatitis. Current evidence suggests that patients are best served by delaying interventions for at least 4 weeks, draining as a first resort, and debriding recalcitrant tissue using minimally invasive techniques to promote or enhance postoperative recovery while reducing wound-related complications.

Keywords: necrotizing pancreatitis, pancreatic necrosectomy, VARD, pancreatic debridement, pancreatic collections

\section{Introduction}

Acute pancreatitis is the leading gastrointestinal cause of hospitalization in the US, with 270,000 admissions annually, and its incidence continues to increase year-over-year. ${ }^{1}$ The economic burden of pancreatitis is estimated at $\$ 2.5$ billion per year. ${ }^{2}$ The personal burden of acute pancreatitis includes debilitating pain and potentially profound morbidity and mortality. Fortunately, in the majority of cases, acute pancreatitis manifests as a mild, self-limited course with few to no long-term sequela. However, in $15 \%-25 \%$ of patients, it manifests with tissue necrosis or infection. This form of the disease is associated with serious complications in $10 \%-30 \%$ of patients, ${ }^{3}$ such as endocrine and exocrine pancreatic insufficiency, organ failure, fistulae, bleeding, and death.

This review covers the pathophysiology, diagnosis, and management of acute pancreatitis. In particular, it addresses challenges and solutions to the management of necrotizing pancreatitis amidst the changing landscape of multidisciplinary and increasingly minimally invasive interventions. This review is based upon a PubMed ${ }^{\circledR}$ search of English language clinical articles and guidelines published within the last 5 years plus those of historic significance; it also provides commentary on technique from our senior authors.

\section{Pathophysiology and classification}

Inappropriate activation of pancreatic proenzymes within the gland itself leads to tissue and microvascular injury, release of pro-inflammatory mediators, and local inflammation. If the release of these mediators is robust enough, the patient develops a systemic inflammatory response. Notably, anti-inflammatory cytokines are also 
produced and may actually overcompensate and inhibit the immune response, rendering the host at risk for systemic infection. ${ }^{4}$ Moreover, it has been shown that activation of nuclear factor-kappa B (NF- $\kappa \mathrm{B})$ links the initial injury to systemic inflammation. ${ }^{5}$ Its early intra-acinar activation and continuous importance in mediating the expression of numerous genes involved in inflammation suggest that inhibiting the activity of NF- $\mathrm{B}$ may prove to be useful. Such targeted inhibition of NF- $\mathrm{KB}$ has shown promise with various cancers and other inflammatory processes, thus may have profound effects in acute pancreatitis. ${ }^{5}$ Table 1 lists various risk factors for pancreatitis, of which gallstones and alcohol consumption are the most common.

A comprehensive classification system is paramount for achieving consistency across studies and reliable communication between clinicians. The Atlanta classification (1992) and its most recent revision (2012) attempted to account for the natural variation in pancreatitis by subdividing it along several different etiologies. The revised version of the Atlanta classification updated and categorized the types of pancreatitis into interstitial edematous pancreatitis and acute necrotizing pancreatitis (ANP). Further subdivision of necrotizing pancreatitis includes parenchymal necrosis alone, peripancreatic necrosis alone, and a combined type with or without infection. ${ }^{6}$

While the Atlanta classification scheme was criticized for its broadness, the recently published IAP/APA (International Association of Pancreatology/American Pancreatic Association) guidelines provide concise evidencebased recommendations regarding the medical and surgical management of pancreatitis. ${ }^{7}$ The recommendations in the IAP/APA guidelines address 38 clinically relevant questions regarding diagnosis, prognostication, imaging, fluid therapy,

Table I Risk factors for pancreatitis

\begin{tabular}{l} 
Anatomic and functional disorders \\
Pancreas divisum \\
Sphincter of Oddi dysfunction \\
Periampullary tumors \\
Autoimmune (eg, systemic lupus erythematosus) \\
Gallstones \\
Hypertriglyceridemia \\
Hypercalcemia \\
Infections and parasitic organisms \\
Toxins \\
Alcohol \\
Medications \\
Rare animal bites \\
Trauma (including iatrogenic post-procedural) \\
Vasculitis \\
\hline Note: Data from Mitchell et al. ${ }^{25}$
\end{tabular}

intensive care management, infection control, nutritional support, and interventions. Many of these recommendations are incorporated into this review; however, a detailed discussion of each point is beyond the scope of this article.

\section{Clinical course}

Most episodes of acute pancreatitis are mild and only necessitate a short hospitalization $(\sim 48 \mathrm{~h})$. Mild acute pancreatitis is characterized by the absence of organ failure and/or pancreatic necrosis. The severe form of disease, however, has a distinct two-phase course: 1) an early phase (within 1-2 weeks) marked by the systemic inflammatory response syndrome (SIRS) and/or organ failure, and 2) a late phase (after 2 weeks) marked by local complications, such as evolving pancreatic and peripancreatic necrosis (sterile or infected), and peripancreatic fluid collections such as pseudocysts. ${ }^{2}$

\section{Diagnosis}

Acute pancreatitis generally presents with sudden onset of upper abdominal pain, usually associated with nausea and vomiting. Pain may radiate to the back. Patients are usually restless and bend forward (knee-chest position) to relieve the pain. ${ }^{8}$ The IAP/APA guidelines define acute pancreatitis based on the fulfillment of two out of three criteria: clinical signs/symptoms (upper abdominal pain), laboratory values (serum amylase or lipase $>3 \times$ upper limit of normal), and/ or imaging findings (ultrasound/computed tomography [CT]/ magnetic resonance imaging [MRI]). ${ }^{7}$ No single laboratory value is predictive of acute pancreatitis, but amylase and lipase - although not part of the revised Atlanta model - are preferred for staging and diagnosis. ${ }^{6}$

Multiple scoring systems such as Acute Physiology and Chronic Health Evaluation II and Ranson criteria are designed for risk stratification. These often rely on complex and poorly recalled criteria, including the hematocrit, imaging, blood urea nitrogen, serum creatinine, serum lactate dehydrogenase, serum urinary trypsinogen, and cytokines. Although there is no data to conclude that any one method is clearly superior, persistence of the SIRS may be the most practical prognosticator. Of note, timing is important with respect to inflammatory laboratory values as values may peak or mature over a period of days and not accurately reflect the patient's current condition. ${ }^{2}$

\section{Medical management}

The first-line intervention for ANP is intravenous hydration with isotonic and $\mathrm{pH}$-balanced crystalloid solution such as Lactated Ringer's (LR). ${ }^{7,9,10}$ At least one randomized trial 
has discovered that goal-directed fluid resuscitation based on changes in BUN vs clinical assessments results in no difference in volume of resuscitation or SIRS. However, this trial did uncover a lower rate of SIRS after resuscitation with LR as opposed to normal saline. ${ }^{10}$ In the past, patients with acute pancreatitis were kept NPO in order to give rest to the pancreas. That rule is no longer strictly adhered to insofar as in mild pancreatitis prompt resumption of oral intake has been shown to result in shorter hospital stays. Total parenteral nutrition is avoided in mild acute pancreatitis as enteral nutrition has been proven to reduce infectious complications, organ failure, and mortality. Routine use of prophylactic antibiotics with severe acute pancreatitis is not recommended as there has been no definitive supportive data. ${ }^{2}$ A portion of patients with acute pancreatitis may experience vascular complications such as splenic, portal, or mesenteric venous thrombosis or arterial pseudoaneurysm and varices. In fact, splenic vein thrombosis $(1 \%-24 \%)$ is a well-recognized complication, but the focus of treatment should remain on pancreatitis as spontaneous resolution has been documented. ${ }^{11}$

For patients who fail to improve, repeat CT or MRI should be considered, although there are varied opinions on the timing. Early CT scanning is not recommended as it does not influence treatment or improve clinical outcomes above and beyond clinical assessment; furthermore, CT scans are not without risks such as contrast reactions. ${ }^{7}$ Optimum time for initial CT is agreed to be $72-96 \mathrm{~h}^{7}$ When invasive intervention is considered, CT and MRI are preferred as the diagnostic modalities.

\section{Interventional challenges}

Perhaps the most challenging aspect of managing acute pancreatitis is deciding whether, when, and how to escalate from supportive care to some type(s) of intervention. There are numerous recent reviews, including our own, which describe this escalation of care in detail. ${ }^{2,3}$ Briefly, the basic principles in this regard are to 1) utilize a multidisciplinary team of radiologists, interventional endoscopists, intensivists, and surgeons; 2) delay-drain-debride (3Ds); and 3) avoid open surgery.

\section{Indications and timing of intervention}

To reiterate, the clinical course of ANP can be divided into two phases. The first phase (ie, 1-2 weeks from symptom onset) is marked by a systemic inflammatory response. Although patients appear quite ill during this phase with significant risk of multiple organ failure, the best treatment is still aggressive supportive care. Consensus guidelines recommend reserving intervention for exceptional complications, such as abdominal compartment syndrome, infected necrosis causing sepsis, and bowel ischemia. During the second phase indications for intervention broaden to include infected necrosis regardless of sepsis and symptomatic sterile necrosis (eg, disabling pain, failure to thrive, gastric or biliary obstruction, and bleeding). ${ }^{12}$

These guidelines also recommend deferring drainage or debridement for 4-6 weeks from symptom onset to allow for demarcation and encapsulation. This mitigates the risk of bleeding, perforation, and loss of vital tissue. A retrospective study of open operative treatment for infected necrosis with an unusually high overall mortality rate nonetheless reported significantly improved mortality as surgery was further deferred $(75 \% \leq 14$ days vs $8 \% \geq 30$ days $) .{ }^{13}$ This same study included a meta-analysis of 11 reports with 1,136 patients confirming that postponing surgery in stable patients for at least 30 days reduced mortality but increased antibiotic use and fungal superinfection. A more recent study that evaluated multiple types of intervention and adjusted for baseline factors and disease severity also found that mortality declined with time to intervention (odds ratio [OR] 0.38, confidence interval [CI] $0.24-0.59, P<0.001) .{ }^{14}$

However, to defer is not to ignore, and patients should undergo less invasive intervention(s) as needed to bridge to or obviate more invasive surgical interventions. Likewise, although a subset of patients with infected necrosis will recover with antibiotics alone, patients with signs of deterioration warrant immediate intervention. Indeed, as a word of caution, many of the studies that flaunt lower mortality with noninterventional treatment are subject to significant selection bias with sicker patients in their interventional cohorts. ${ }^{14,15}$

\section{Minimize invasiveness}

Once the decision has been made to intervene, there are a number of options theoretically available to the clinician. The traditional approach is open necrosectomy with closed packing, ${ }^{16}$ repeat debridement (Figure 1), or continuous lavage. Modern alternatives include laparoscopic transperitoneal necrosectomy, ${ }^{17}$ video-assisted retroperitoneal debridement (VARD) (Figure 2), ${ }^{18}$ endoscopic necrosectomy, ${ }^{19}$ percutaneous drainage, and step-up or hybrid approaches. ${ }^{20,21}$

Based on a cadre of randomized clinical trials and prospective studies, the treatment paradigm has shifted from open surgery to a minimally invasive, step-up algorithm., ${ }^{2,12}$ Radiologically guided percutaneous drainage should be 
the first step. The PANTER trial demonstrated that a stepup approach from percutaneous drainage yields lower complications, especially hernia and new-onset diabetes, compared to primary open necrosectomy. ${ }^{20}$ If percutaneous drainage appears inadequate after optimization of drain size and position, the clinician should advance to debridement by endoscopic or laparoscopic (usually retroperitoneal) techniques. The PENGUIN trial demonstrated that endoscopic necrosectomy induces less inflammation as measured by the levels of interleukin- 6 and yields lower morbidity and mortality compared to primary surgical necrosectomy. ${ }^{19}$ The TENSION trial currently underway in the Netherlands is intended to assess the optimal way to perform a minimally invasive step-up approach (endoscopic vs videoscopic necrosectomy). ${ }^{22}$ What is certain is that open surgery should be a last resort at best.

\section{Remaining challenges and solutions}

Despite the availability of randomized trials, much remains to be validated, substantiated, and answered. This was made evident in a recent Cochrane Review of eight RCTs with 306 patients focused on the treatment of necrotizing pancreatitis. ${ }^{23}$ The review underscored the very low quality of evidence due to small sample sizes, inherent biases, and inconsistent outcomes. Moreover, the authors noted the absence of longterm mortality, quality of life, or return to work outcomes.

Another major challenge that is yet to be addressed is the creation and validation of better predication models. Knowing who is at risk of pancreatitis, necrotizing disease, and infection or organ failure could allow for preventative measures or better direct management. Even if intervention cannot be obviated, perhaps clinicians could at least pare down from a serial or step-up treatment to a single-stage definitive procedure.

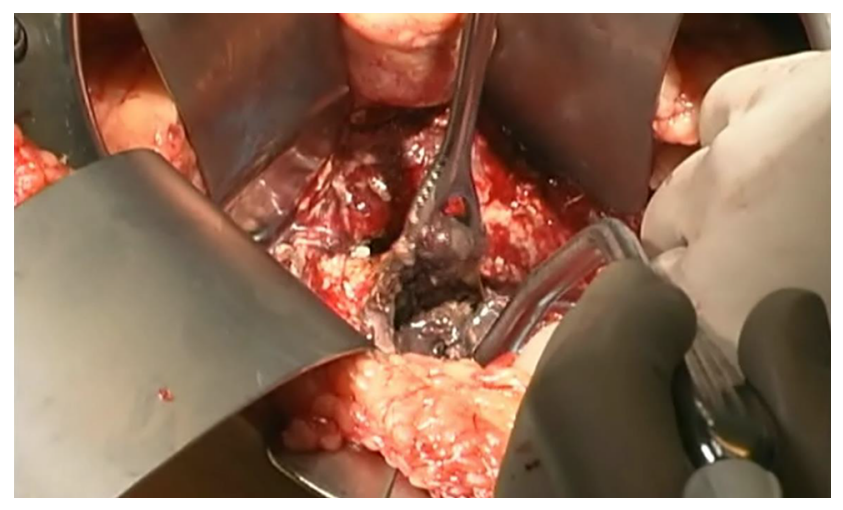

Figure I Open debridement.

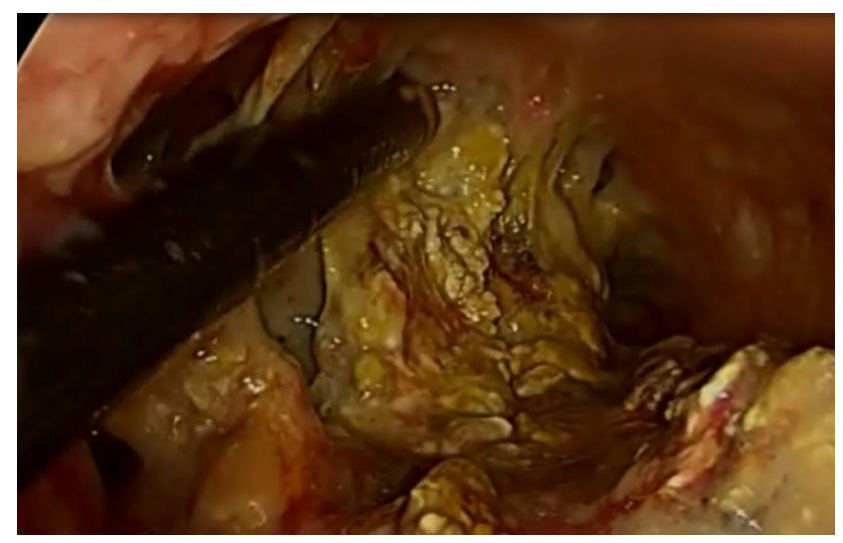

Figure 2 Video-assisted retroperitoneal debridement

Another model should be created to determine which approach is best for a given patient. For instance, $\sim 50 \%$ of patients undergoing percutaneous drainage will progress to more aggressive treatment. ${ }^{2}$ In a retrospective analysis of 130 patients undergoing percutaneous drainage of pancreatic necrosis, male sex, multiorgan failure (OR 0.15, CI 0.04 $0.62, P<0.01)$, increasing percentage of pancreatic necrosis, and heterogeneity of collection were negative predictors of success (ie, survival without operative necrosectomy). Oddly, despite the apparent likelihood of failure, given these factors, the authors still advocate step-up approach in all patients. ${ }^{24}$ Similarly, of those who are considered for endoscopic treatment, $30 \%$ will not be candidates due to technical issues. ${ }^{18}$

\section{Nuances of surgical drainage and debridement}

In the absence of rigorous trials about the nuances of drainage and debridement, we offer our own algorithm on this topic. To begin with, the approach should be dictated by the location of the necrotic segment. For necrosis in the tail, a simple retroperitoneal approach is often appropriate either with radiological guidance for percutaneous drainage or along a drain tract for surgical debridement. A single-incision laparoscopy port or one 5 -mm port placed alongside a $12-\mathrm{mm}$ port can help improve utilization of the narrow space during trans-retroperitoneal debridement. Alternatively, mid-gland necrosis with a viable pancreatic tail will require long-term drainage either internally into the stomach or small intestine. Drainage into the stomach is usually the most convenient method because of its proximity and it allows for subsequent endoscopic evaluation and debridement if necessary. This can be accomplished through a laparoscopic transabdominal approach through 
whichever of the gastrocolic ligament or mesocolon is less inflamed. Although lesions of the head can also be drained internally to adjacent bowel or a roux limb, such procedures are exceedingly complex and fraught with the risk of bleeding or leakage. In general, bleeding from a necrotic pancreas is best addressed with packing immediately followed by coiling in interventional radiology. Regardless of the approach, blunt dissection is recommended for initial debridement of the necrosum; the suction irrigator is often ideal in this regard and can delineate vital from nonvital tissue. The use of laparoscopic instruments such as the $10-\mathrm{mm}$ stone extractor to facilitate the efficient removal of larger pieces of necrotic debris was then recommended. Ultimately, when a round of debridement is completed, large bore drains should be left in place to promote a pathway for postoperative irrigation and drainage and also to facilitate future intervention if needed.

\section{Conclusion}

When acute pancreatitis becomes necrotizing pancreatitis invasive treatment becomes more likely. The most recent data favor minimally invasive techniques, but robust studies are yet to delineate if and when one option is superior to another one. There are also many nuances to the drainage and debridement of necrotic pancreas outside the scope of available or pending studies.

\section{Disclosure}

The authors report no conflicts of interest in this work.

\section{References}

1. Peery AF, Dellon ES, Lund J, et al. Burden of gastrointestinal disease in the United States: 2012 update. Gastroenterology. 2012;143(5):11791187.e1-3.

2. Tenner S, Baillie J, DeWitt J, Vege SS. American College of Gastroenterology. American College of Gastroenterology guideline: management of acute pancreatitis. Am J Gastroenterol. 2013;108(9):1400-1415;1416.

3. Kokosis G, Perez A, Pappas TN. Surgical management of necrotizing pancreatitis: an overview. World J Gastroenterol. 2014;20(43): 16106-16112.

4. Kylanpaa L, Rakonczay Z Jr, O'Reilly DA. The clinical course of acute pancreatitis and the inflammatory mediators that drive it. Int $J$ Inflam. 2012;2012:360685.

5. Jakkampudi A, Jangala R, Reddy BR, Mitnala S, Reddy DN, Talukdar R. NF-kappaB in acute pancreatitis: mechanisms and therapeutic potential. Pancreatology. 2016;16(4):477-488.
6. Thoeni RF. The revised Atlanta classification of acute pancreatitis: its importance for the radiologist and its effect on treatment. Radiology. 2012;262(3):751-764.

7. Working Group IAP/APA Acute Pancreatitis Guidelines. IAP/APA evidence-based guidelines for the management of acute pancreatitis. Pancreatology. 2013;13(4 Suppl 2):e1-e15.

8. Carroll JK, Herrick B, Gipson T, Lee SP. Acute pancreatitis: diagnosis, prognosis, and treatment. Am Fam Physician. 2007;75(10):1513-1520.

9. Buxbaum J, Yan A, Yeh K, Lane C, Nguyen N, Laine L. Aggressive hydration with lactated Ringer's solution reduces pancreatitis after endoscopic retrograde cholangiopancreatography. Clin Gastroenterol Hepatol. 2014;12(2):303-307. e301.

10. Wu BU, Hwang JQ, Gardner TH, et al. Lactated Ringer's solution reduces systemic inflammation compared with saline in patients with acute pancreatitis. Clin Gastroenterol Hepatol. 2011;9(8):710-717. e711.

11. Nadkarni NA, Khanna S, Vege SS. Splanchnic venous thrombosis and pancreatitis. Pancreas. 2013;42:924.

12. Freeman ML, Werner J, van Santvoort HC, et al. Interventions for necrotizing pancreatitis: summary of a multidisciplinary consensus conference. Pancreas. 2012;41(8):1176-1194.

13. Besselink MG, Verwer TJ, Schoenmaeckers EJ, et al. Timing of surgical intervention in necrotizing pancreatitis. Arch Surg. 2007;142(12): 1194-1201.

14. van Santvoort HC, Bakker OJ, Bollen TL, et al. A conservative and minimally invasive approach to necrotizing pancreatitis improves outcome. Gastroenterology. 2011;141(4):1254-1263.

15. Mouli VP, Sreenivas V, Garg PK. Efficacy of conservative treatment, without necrosectomy, for infected pancreatic necrosis: a systematic review and meta-analysis. Gastroenterology. 2013;144(2):333-340.e2.

16. Rodriguez JR, Razo AO, Targarona J, et al. Debridement and closed packing for sterile or infected necrotizing pancreatitis: insights into indications and outcomes in 167 patients. Ann Surg. 2008;247:294-299.

17. Parekh D. Laparoscopic-assisted pancreatic necrosectomy: a new surgical option for treatment of severe necrotizing pancreatitis. Arch Surg. 2006;141:895-902.

18. Gomatos IP, Halloran CM, Ghaneh P, et al. Outcomes from minimal access retroperitoneal and open pancreatic necrosectomy in 394 patients with necrotizing pancreatitis. Ann Surg. 2016;263(5):992-1001.

19. Bakker OJ, van Santvoort HC, van Brunschot S, et al. Endoscopic transgastric vs surgical necrosectomy for infected necrotizing pancreatitis: a randomized trial. JAMA. 2012;307:1053-1061.

20. van Santvoort HC, Besselink MG, Bakker OJ, et al. A step-up approach or open necrosectomy for necrotizing pancreatitis. $N$ Engl $J$ Med. 2010;362:1491-1502.

21. van Brunschot S, Bakker OJ, Besselink MG, et al. Treatment of necrotizing pancreatitis. Clin Gastroenterol Hepatol. 2012;10(11):1190-1201.

22. van Brunschot S, van Grinsven J, Voermans RP, et al. Transluminal endoscopic step-up approach versus minimally invasive surgical step-up approach in patients with infected necrotising pancreatitis (TENSION trial): design and rationale of a randomised controlled multicenter trial [ISRCTN09186711]. BMC Gastroenterol. 2013;13:161.

23. Gurusamy KS, Belgaumkar AP, Haswell A, et al. Interventions for necrotising pancreatitis. Cochrane Database Syst Rev. 2016;4:CD011383.

24. Hollemans RA, Bollen TL, van Brunschot S, et al. Predicting success of catheter drainage in infected necrotizing pancreatitis. Ann Surg. 2016; 263(4):787-792.

25. Mitchell RM, Byrne MF, Baillie J. Pancreatitis. Lancet. 2003;361(9367): $1447-1455$. 


\section{Publish your work in this journal}

Clinical and Experimental Gastroenterology is an international, peerreviewed, open access, online journal publishing original research, reports, editorials, reviews and commentaries on all aspects of gastroenterology in the clinic and laboratory. This journal is included on PubMed. The manuscript management system is completely online and includes a very quick and fair peer-review system, which is all easy to use. Visit http://www.dovepress.com/testimonials.php to read real quotes from published authors.

Submit your manuscript here: https://www.dovepress.com/clinical-and-experimental-gastroenterology-journal 